\title{
Peptoid nanosheets exhibit a new secondary-structure motif
}

Ranjan V. Mannige ${ }^{1}$, Thomas K. Haxton ${ }^{1}$, Caroline Proulx ${ }^{1}$, Ellen J. Robertson ${ }^{1}$, Alessia Battigelli ${ }^{1}$, Glenn L. Butterfoss ${ }^{2}$, Ronald N. Zuckermann ${ }^{1} \&$ Stephen Whitelam $^{1}$

A promising route to the synthesis of protein-mimetic materials that are capable of complex functions, such as molecular recognition and catalysis, is provided by sequence-defined peptoid polymers ${ }^{1,2}$ structural relatives of biologically occurring polypeptides. Peptoids, which are relatively non-toxic and resistant to degradation ${ }^{3}$, can fold into defined structures through a combination of sequencedependent interactions ${ }^{3-8}$. However, the range of possible structures that are accessible to peptoids and other biological mimetics is unknown, and our ability to design protein-like architectures from these polymer classes is limited'. Here we use molecular-dynamics simulations, together with scattering and microscopy data, to determine the atomic-resolution structure of the recently discovered peptoid nanosheet, an ordered supramolecular assembly that extends macroscopically in only two dimensions. Our simulations show that nanosheets are structurally and dynamically heterogeneous, can be formed only from peptoids of certain lengths, and are potentially porous to water and ions. Moreover, their formation is enabled by the peptoids' adoption of a secondary structure that is not seen in the natural world. This structure, a zigzag pattern that we call a
$\Sigma$ ('sigma')-strand, results from the ability of adjacent backbone monomers to adopt opposed rotational states, thereby allowing the backbone to remain linear and untwisted. Linear backbones tiled in a brick-like way form an extended two-dimensional nanostructure, the $\Sigma$-sheet. The binary rotational-state motif of the $\Sigma$-strand is not seen in regular protein structures, which are usually built from one type of rotational state. We also show that the concept of building regular structures from multiple rotational states can be generalized beyond the peptoid nanosheet system.

The peptoid nanosheet is a recently discovered, free-floating planar assembly that is only two molecules thick but that extends laterally for micrometres (Supplementary Figs 1,2) ) $^{8,10,11}$. Nanosheets assemble from peptoids bearing alternating aromatic and charged sidechains (Fig. 1a) via compression at an air-water interface ${ }^{8,10-12}$. We find, through a combination of atomic-force microscopy (AFM; Supplementary Fig. 3$)^{8,10,13}$ and powder X-ray diffraction $(\mathrm{XRD})^{8,10,11,13}$, that nanosheets are bilayers, $3.0 \pm 0.3 \mathrm{~nm}$ ( \pm s.d.) thick, in whose interior the aromatic sidechains are sequestered, and on whose surfaces the charged sidechains are presented. Optical microscopy shows nanosheets to be

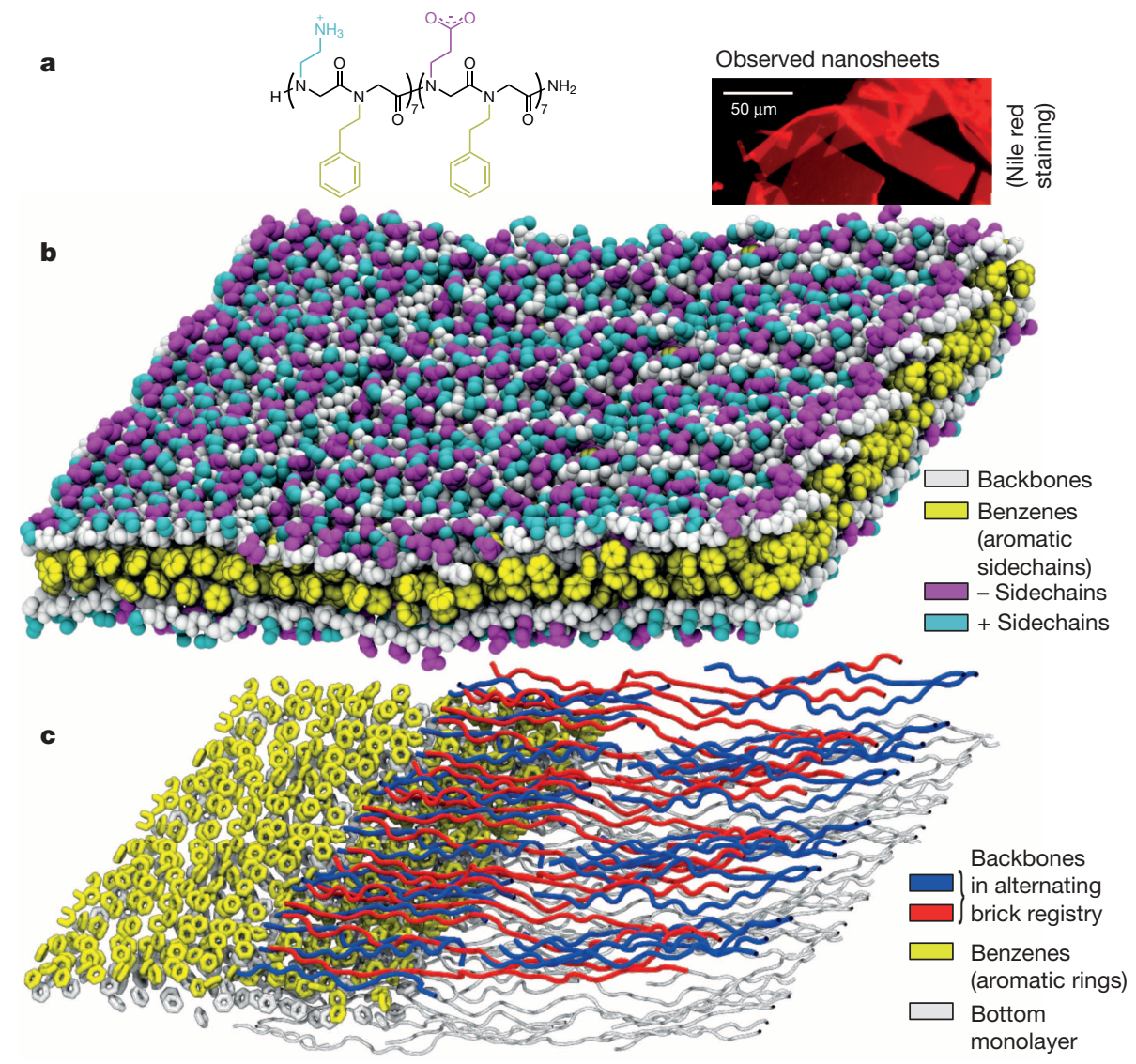

Figure 1 Snapshot of a peptoid nanosheet obtained from molecular-dynamics simulations. a, Left, an amphiphilic 28-residue peptoid, which assembles into extended nanosheets only two molecules thick ${ }^{10}$, as shown in the fluorescentmicroscopy image to the right. $\mathbf{b}$, Snapshot of a bilayer, obtained from molecular-dynamics simulations. c, Backbones (blue and red) are generally collinear; aromatic rings (yellow) in the interior of the nanosheet show little order. (The lower leaf of the bilayer is coloured white.)

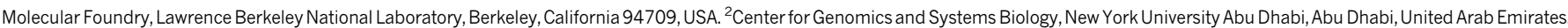


roughly rectangular, many square micrometres in horizontal extent, and to have relatively straight edges (Fig. 1a and Supplementary Fig. 2b). XRD measurements in the plane of the bilayer show peaks at characteristic distances of $4.5 \AA$ and $3.6 \AA$ (Supplementary Fig. 9), suggesting a high degree of order on the molecular scale. Direct observation of polymer chains in the nanosheet by transmission electron microscopy indicates a polymer-polymer parallel spacing of about $4.5 \AA$ (ref. 8), in accordance with the 4.5 - $\mathrm{XRD}$ peak.

To develop an atomistic model of peptoid nanosheets consistent with these observations, we used atomistic molecular-dynamics simulations in conjunction with our recently developed CHARMMbased $^{14}$ force field for peptoid backbones, MFTOID ${ }^{15}$ (CHARMM, Chemistry at HARvard Molecular Mechanics; MFTOID, Molecular Foundry (MF) and Peptoid (TOID)). We surveyed a range of lowenergy nanosheet configurations (Supplementary Fig. 4a) as starting points for molecular-dynamics simulations; here, we report the results of one such set of simulations (see Methods).

Figure $1 \mathrm{~b}$ and $\mathrm{c}$ show snapshots of a nanosheet patch, with periodic images displayed (for an image of the simulation box only, see Supplementary Fig. 4c). The nanosheet possesses linear order in its

a Viewpoints

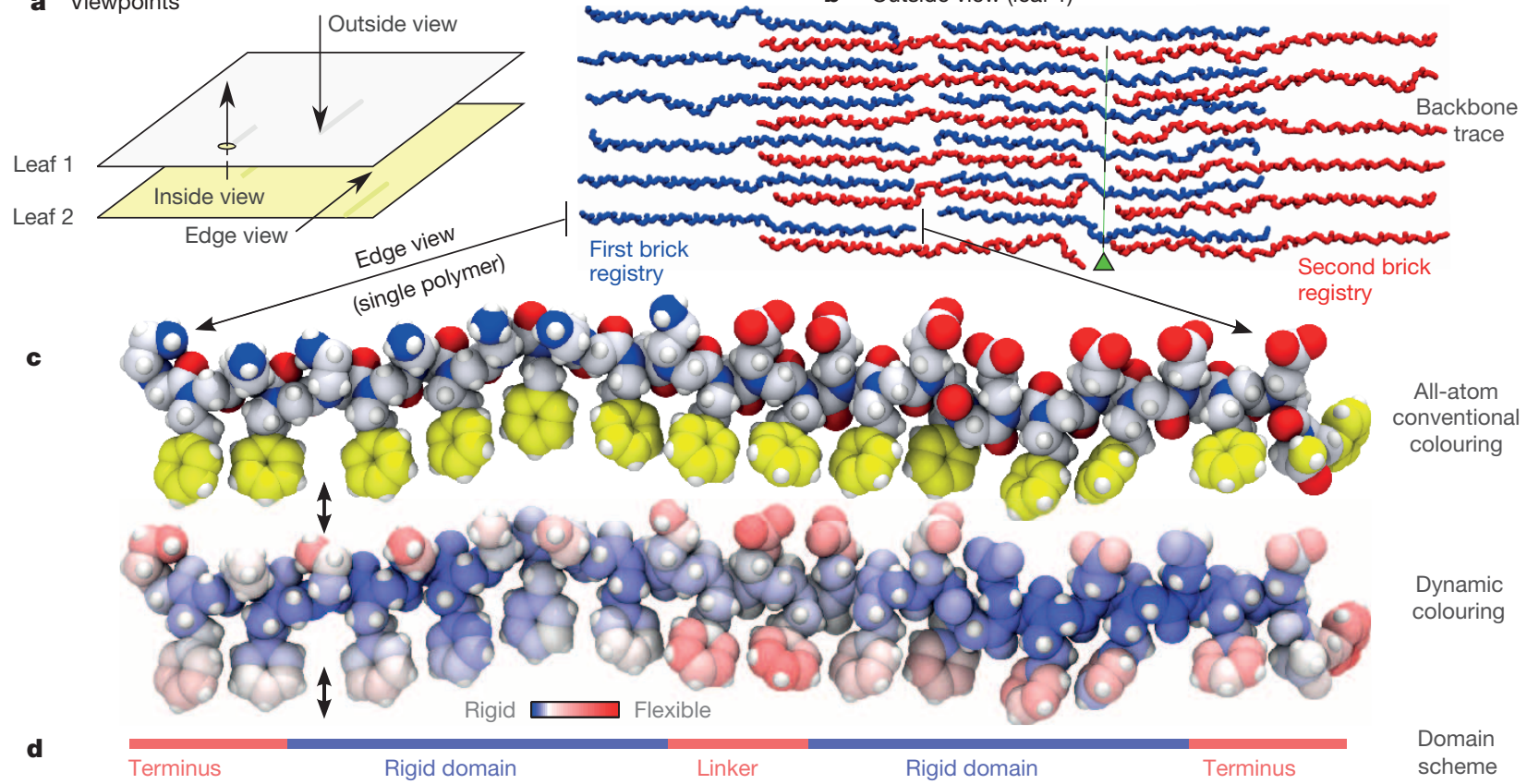

b Outside view (leaf 1) 
polymer backbones, and relative disorder in the aromatic and charged sidechains that are, respectively, internal and external to the bilayer (Supplementary Figs 11 and 12). Individual peptoid chains maintain the brick-like arrangement that is favoured by the segregation of charged sidechains into positively and negatively charged blocks (Fig. 1a), in which gaps or 'pockets' between polymer termini alternate with the backbones' central portions, as one looks along the direction perpendicular to that of the polymer chains (Fig. 2b). As shown in Fig. 2c and Supplementary Fig. 12, backbone regions away from the pockets are the least dynamic portions of each peptoid, displaying a root-mean-squared fluctuation (RMSF) of $\leq 1 \AA$. The pocket-forming termini of each peptoid are more flexible than these interior regions, and are visibly less ordered (Fig. 2c, d and Supplementary Fig. 12), while the polymer sidechains are more flexible still. This hierarchy of flexibility is similar to that seen in proteins ${ }^{16,17}$.

Figure $2 \mathrm{e}-\mathrm{g}$ and Supplementary Fig. 8 show that the nanosheet thickness, interpolymer spacings and intersidechain spacings seen in our simulations lie within about an ångström of the mean characteristic distances seen in AFM and X-ray-scattering experiments ${ }^{8,10,11}$.
The range of nanosheet thicknesses measured by AFM, $2.7 \mathrm{~nm}$ to $3.3 \mathrm{~nm}$ (Supplementary Fig. 3), is also reproduced in our simulations (Supplementary Fig. 8). These comparisons allow us to assign physical features to experimental measurements, and to verify the accuracy of our simulations (see Supplementary Table 1).

This brick-like arrangement of backbones that allows polymers to alternate with pockets has important consequences for the stability of nanosheets as a function of peptoid length. As polymer length decreases, it should become less energetically favourable for each residue to form a nanosheet, because pockets-near which peptoids possess fewer favourable electrostatic and aromatic interactions-increase in number per unit area as polymer length decreases. Simulations indeed show that short polymers are not stable in nanosheet form. In Fig. 3, we report the results of simulations done using nanosheets built from polymers that are 4,8 , $12,16,20,24$ or 28 residues long. Polymers longer than 12 residues form stable nanosheets. Nanosheets built from 12-residue polymers show signs of instability and a decrease in order (Fig. $3 \mathrm{a}-\mathrm{c}$ ) upon simulation, and nanosheets built from polymers shorter than 12 residues display an almost complete loss of structure within 40 ns of the start of the simu-

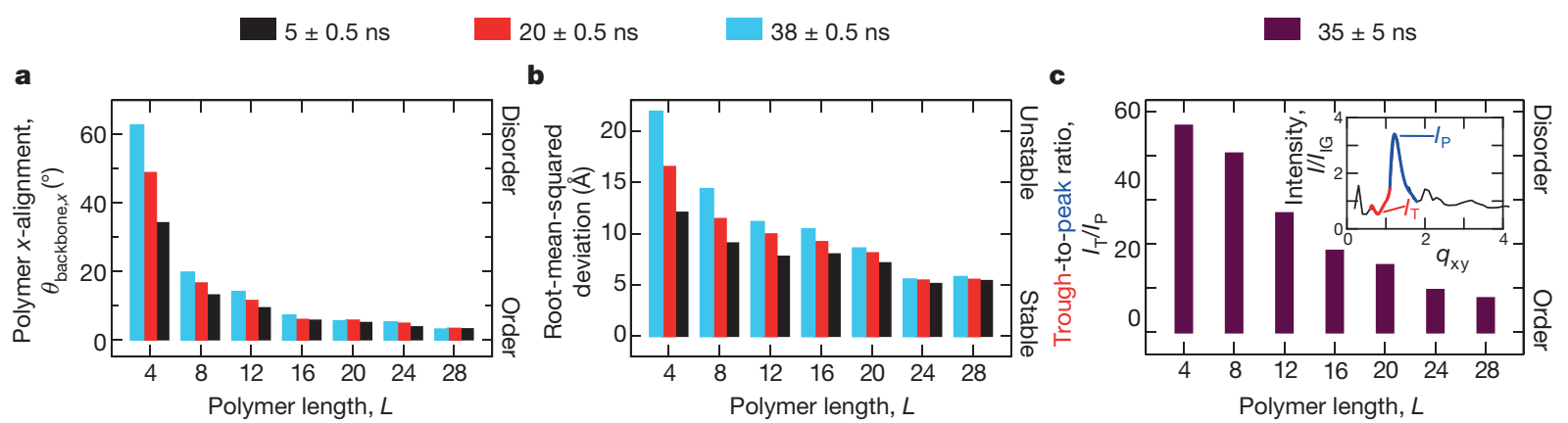

d
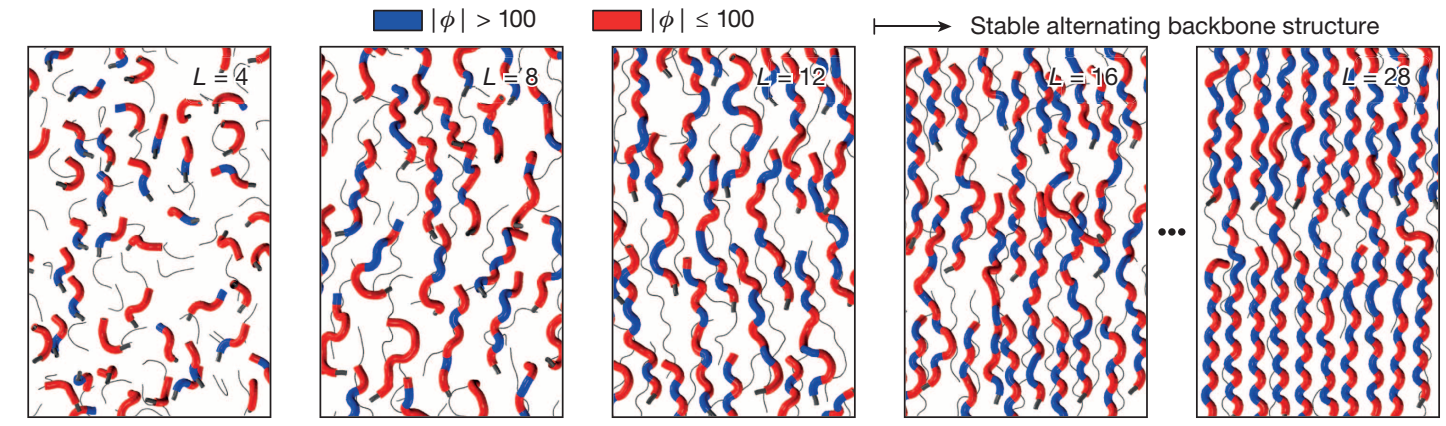

e
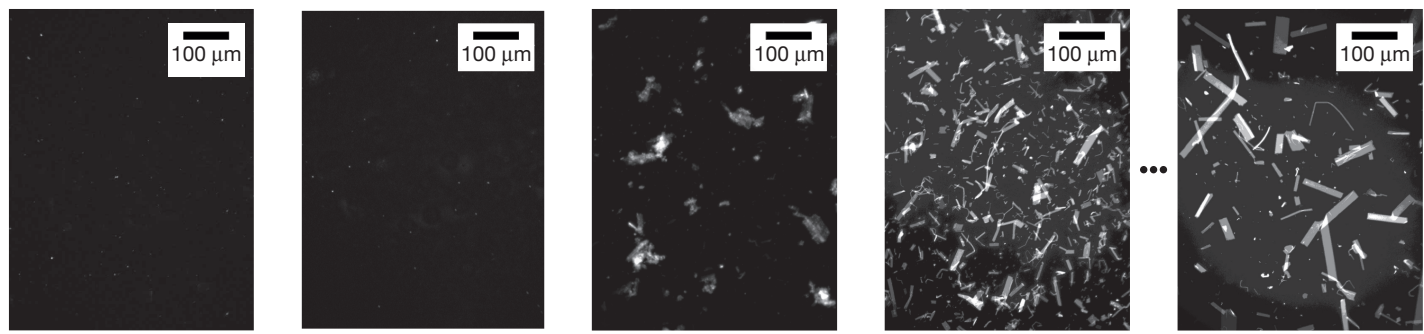

$\rightarrow$ Stable nanosheets from experiment

4

8

12

16

28

Polymer length, $L$

Figure $3 \mid$ Peptoids shorter than 12 residues do not form stable nanosheets. a-c, Metrics of stability and order for nanosheets built from peptoids of various lengths, $L$. These metrics are: a, mean polymer alignment with the $x$ axis (equation (2) in Methods; see Supplementary Fig. 16a); b, root-mean-squared deviation from the initial configuration (heavy atoms only; see Supplementary Fig. 16b); and c, trough-to-peak ratio associated with backbone-backbone order obtained from simulated X-ray scattering (a spectrum for $L=28$ is shown in the inset; see Supplementary Fig. 17). Larger values of all metrics indicate a lesser degree of order. Short-polymer nanosheets continue to evolve throughout the times simulated $(\mathbf{a}, \mathbf{b}) . I$, intensity; $I_{\mathrm{P}}$, peak intensity; $I_{\mathrm{T}}$, trough intensity; $I_{\mathrm{IG}}$, intensity expected of an ideal gas (for the purposes of normalization); $q_{x y}, x y$ component of the wavevector. $\mathbf{d}, \mathbf{e}$, Simulations (d) and experiments (fluorescence optical microscopy; e) done with peptoids of lengths $L=4,8,12,16$ or 28 show a similar trend: peptoids shorter than 12 residues do not form stable nanosheets. The case $L=12$ is marginal: in experiments, high concentrations of peptoids result in the formation of small nanosheets, which become disordered on a timescale of about one week (see Supplementary Fig. 18). Colouring backbones with respect to the backbone dihedral angle, $\phi$, makes clear that adjacent backbone monomers adopt opposed rotational states. This novel secondary structure, the $\Sigma$-strand, is discussed in Fig. 4 


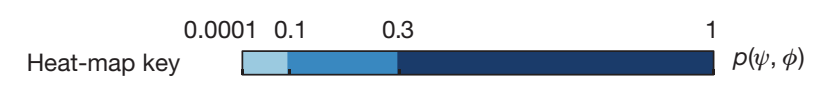

a

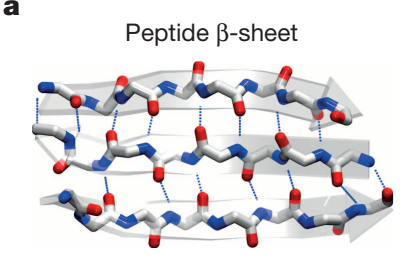

Residue $=\begin{array}{lllll}1 & 2 & 3 & 4 & \cdots\end{array}$

$(\phi, \psi)=i \quad i \quad i \quad i \quad \cdots$

e

Peptoid $\Sigma$-sheet from MD simulation

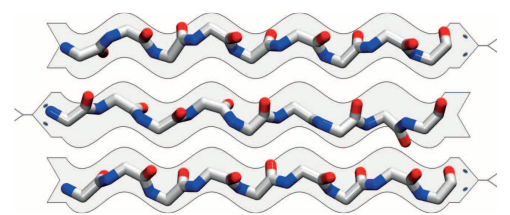

$$
\begin{aligned}
& \text { Residue }=\begin{array}{lllll}
1 & 2 & 3 & 4 & \cdots
\end{array} \\
& (\phi, \psi)=i \quad j \quad i \quad j \quad \ldots
\end{aligned}
$$

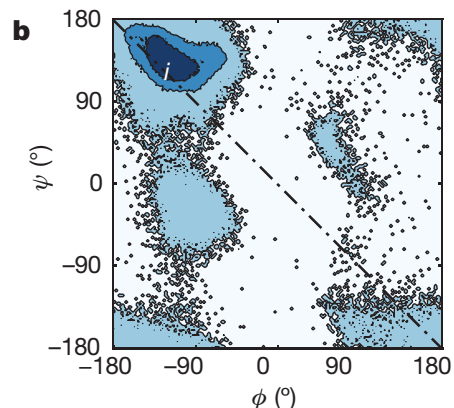

f c

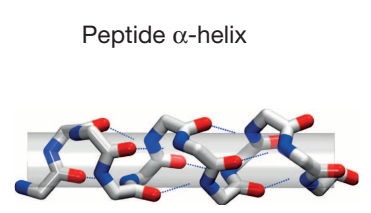

Residue $=\begin{array}{lllll}1 & 2 & 3 & 4 & \cdots\end{array}$

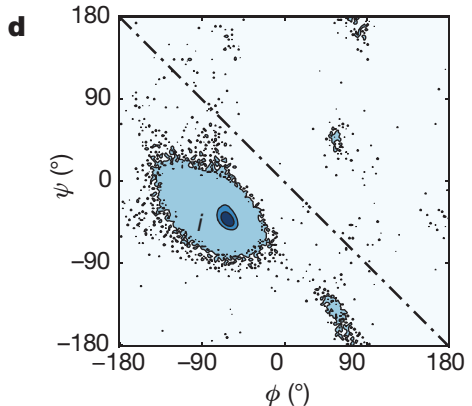

Peptoid $\Sigma$-strand g from QM calculations
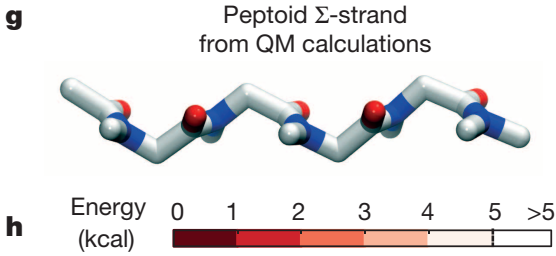

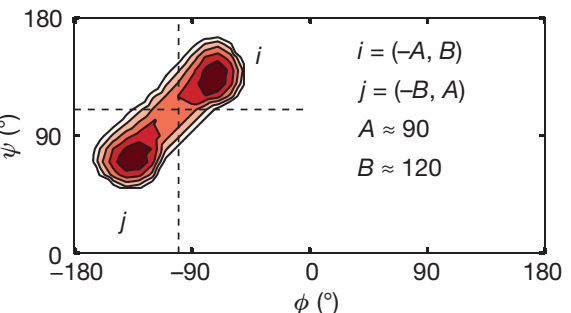

$\phi\left(^{\circ}\right)$

Figure 4 | Peptoid nanosheets are made possible by a novel secondary structure. a-d, Typical secondary structures found in proteins, such as the $\beta$-sheet (a) and $\alpha$-helix (c), are described by specific pairs of backbone dihedral angles, $\phi$ and $\psi$, whose position is labelled by $i$ in their respective

Ramachandran plots (b, d; see Methods). The heat map is a two-dimensional histogram showing the normalized probability $p(\psi, \phi)$ that a residue in one of the indicated structures will adopt particular combinations of the dihedral angles $\phi$ and $\psi$.e, f, In contrast, the secondary structure observed in the peptoid nanosheet (e) consists of two characteristic $(\phi, \psi)$ positions, $i$ and $j(\mathbf{f})$. (The other occupied regions in $\mathbf{f}$, that is, the pale blue locations, indicate polymer ends and linker regions; see Supplementary Figs 21 and 22. Owing to the

lation (Fig. 3d and Supplementary Fig. 15). In accordance with our simulations, our experiments show that peptoids that are 12 or fewer residues in length do not form stable nanosheets (Fig. $3 \mathrm{e}$ and Supplementary Figs 18 and 19). Our simulations suggest that this failure occurs because short-polymer nanosheets are unstable relative to disordered aggregates. Our simulations also suggest that if polymer connections are reinforced-for example, by head-to-tail cross-linking-this limitation could be overcome.

When considering the design of nanosheets, the observation that interchain pockets are tolerated by polymers longer than a certain limit shows that pockets should be considered to be an integral part of the nanosheet structure. Simulations show that such pockets allow water to encroach on the aromatic centre of the bilayer (Fig. 2e and Supplementary Fig. 20b). This observation suggests that peptoid termini could be modified to create pockets able to bind specific small molecules, potentially permitting catalytic function. Furthermore, our simulations show that, if pockets on opposing leaves coincide, then nanosheets possess channels through which water can pass (Supplementary Fig. 20c), indicating the potential of nanosheets as selective membranes. Experimental work to test these predictions is under way.

The stability of extended, planar peptoid nanosheets is enabled by the polymers' linear, untwisted configurations. Our simulations reveal that this linearity results from the ability of sequential backbone residues to adopt one of two states, whose rotation about the backbone axis opposes and cancels each other. In Fig. $3 \mathrm{~d}$ we have coloured these two achirality of the peptoid backbone's $\alpha$-carbon, $i^{\prime}$ and $j^{\prime}$ are equivalent to $i$ and $j$, respectively.) Positions $i$ and $j$ are visibly equidistant from the achiral diagonal of the Ramachandran plot, and so adopting the two states in an alternating fashion allows the peptoid backbone to remain linear and untwisted. This motif, the $\Sigma$-strand, allows the formation of extended planar structures that have not been made previously using protein-like building motifs. g, Quantummechanical (QM) calculations of a range of possible $\Sigma$-type strands show energy minima (h) that match the high-occupancy regions of $\mathbf{f}$, indicating that the rotational tendencies of isolated polymers are preserved by the sidechain interactions established within the nanosheet. (MD, molecular dynamics. The alternating pattern is defined by the numbers $A$ and $B$.)

rotational states red and blue. This building principle is distinct from that used by proteins: the backbones of protein secondary structures such as $\alpha$-helices and $\beta$-sheets are defined primarily by a single rotational state.

Protein rotational states are quantified by their backbone dihedral angles, traditionally denoted $\phi$ and $\psi$, and conventionally described by a Ramachandran plot $^{18}$. As shown in Fig. $4 a-d$, regular chiral protein structures such as the $\alpha$-helix and $\beta$-sheet correspond roughly to a single location, $i$, on the Ramachandran plot $^{18}$. In contrast, stable nanosheets are composed of peptoids whose backbone states occupy two specific regions of the Ramachandran plot (Fig. 4f), labelled $i$ and $j$. Chains whose adjacent residues alternate between these two states remain linear. A snapshot of three backbone segments (Fig. 4e) emphasizes this alternating motif. We call this motif the $\Sigma$-strand, because its linear, twist-free nature derives from the combination, or sum (' $\Sigma$ '), of its two rotational states (and because the resulting polymer 'snakes' back and forth). In principle one could have a $\Sigma$-strand built from any two opposed rotational states. However, density functional theory calculations show that the particular rotational states observed in our atomistic simulations are the lowest-energy $\Sigma$-type arrangement for isolated polymers (Fig. 4g and Supplementary Figs 23-26). This comparison provides additional confidence in the accuracy of the MFTOID force-field simulations, and confirms that the basic rotational tendency of an isolated peptoid backbone ${ }^{15}$ (Fig. $4 \mathrm{~h}$ ) is preserved by the sidechain-sidechain interactions established within the nanosheet (Fig. 4f). 

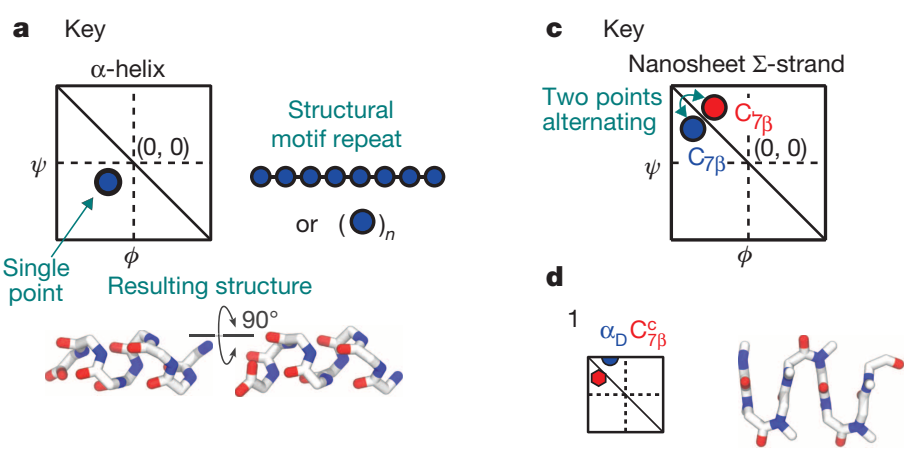

4
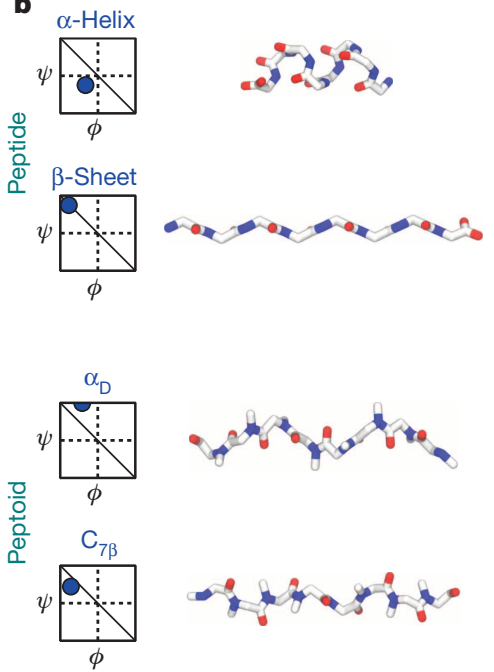

Figure 5 The design principle underlying the peptoid nanosheet has general application. a, b, Canonical secondary structures are 'single-point' ones, meaning that they are described by single regions on a Ramachandran plot. ' $D$ ' and ' $7 \beta$ ' are labels given to certain common peptoid structures. c, Here we propose the concept of building higher-order or multi-point secondary structures using combinations of points (alternating blue and red dots) on the Ramachandran plot. d, This principle can be used to create a large set of novel structures, of which the $\Sigma$-strand is but one example, potentially expanding the range of functional polymer structures. For instance, model 8 shows that a linear chain can display alternating orthogonal sidechains, which may allow the

The $\Sigma$-strand motif is protein-like in its regularity, but is different from protein secondary structures in important ways: it is a molecular motif that permits planar assemblies of macroscopic extent; it is stabilized for reasons other than hydrogen bonding; and it is built from two (not one) rotational states. The closest comparable secondary structures in proteins, $\beta$-sheets, are unable to maintain macroscopic flatness owing to a propeller-like twist in the shape of each strand (although fibril-like protein assemblies can be of considerable size ${ }^{19}$ and can be combined to form structures of macroscopic extent ${ }^{20}$ ). The $\Sigma$-sheet therefore represents a complement to the basic building blocks of the protein world. To replicate the $\Sigma$-sheet assembly using other polymers, one therefore needs two things: molecular rotational properties that give rise to symmetry about the diagonal of the Ramachandran plot (Fig. 4f), and sidechain sequence patterning that promotes these conformations within a desired assembly.

The principle underlying the stability of the $\Sigma$-strand and the peptoid nanosheet is that regular secondary structures can be built from more than one rotational state-that is, from more than one point on the Ramachandran plot. This principle immediately suggests the possibility of a large set of novel secondary structures, some of which are shown in Fig. 5. The potential applications of this set of structures go beyond those of the nanosheet system - the $\Sigma$-strand is but one member of this set-and may include the formation of novel folded structures and

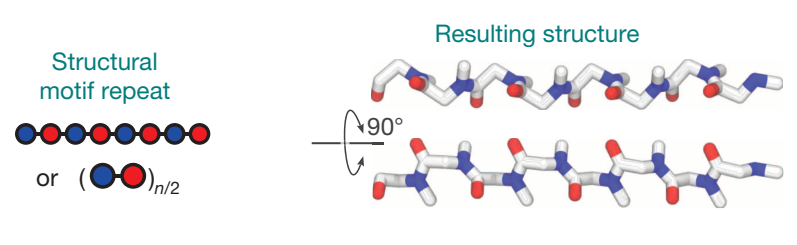

2

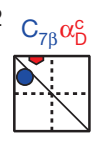

5

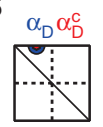

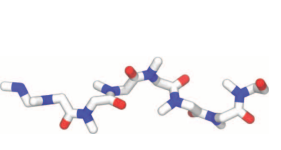

${ }^{3} \alpha_{0} C_{7 \beta}$

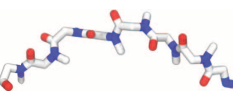

6
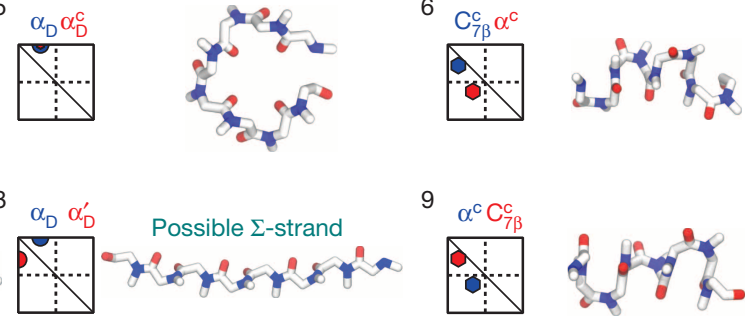

11
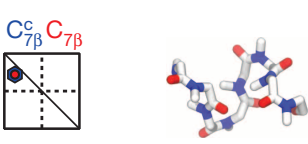

14
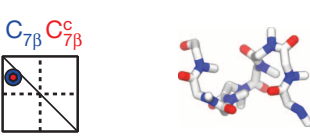

12
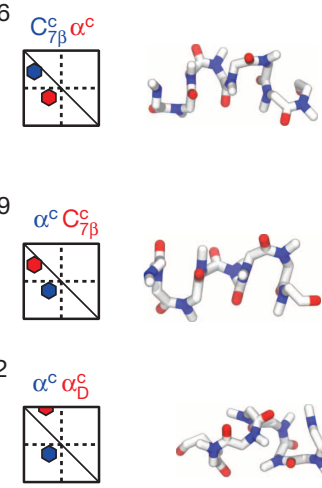

15
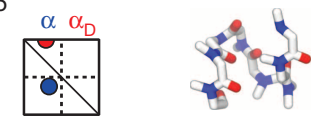

formation of a planar assembly distinct from the $\Sigma$-sheet ('possible $\Sigma$-strand') Model 5, if cyclized, may allow for the assembly of stacked disks (alternating rotational properties have been observed in cyclic peptoid $\mathrm{s}^{22}$, while alternating L,D cyclic peptides have been found to stack ${ }^{23}$ ). As in the case of the $\Sigma$-strand (model 7), such structures can in principle be stabilized by physically reasonable sidechain interactions possessing appropriate geometries. Supplementary Table 2 discusses the method by which the structures in $\mathbf{d}$ were built, relaxed and ranked. For each key in $\mathbf{d}$, the superscript ' $c$ ' indicates a cisamide-bond dihedral angle.

assemblies. Since the 1950s, the literature on protein secondary structure has focused on the idea of building with one type of rotational state $^{18}$, to make 'single-point' Ramachandran structures. We propose that building higher-order, multi-point secondary structures might greatly expand the repertoire of folded polymeric building blocks. Figure $5 \mathrm{~d}$ provides guidelines for backbone design. It may also help to characterize structurally the rapidly growing family of solid-state peptoid polymer crystals ${ }^{21}$. Such crystals consist largely of extended polymer conformations that so far have eluded characterization at atomic resolution; the extended backbones seen in Fig. $5 \mathrm{~d}$ are candidate conformations. More generally, the new building principle that we have identified, combined with the ability to encode into peptoids a defined sequence of chemically diverse monomers, offers a way to create new structured polymers through combinatorial design.

Online Content Methods, along with any additional Extended Data display items and Source Data, are available in the online version of the paper; references unique to these sections appear only in the online paper.

Received 20 April 2015; accepted 27 July 2015.

Published online 7 October 2015.

1. Knight, A. S., Zhou, E. Y., Francis, M. B. \& Zuckermann, R. N. Sequence programmable peptoid polymers for diverse materials applications. Adv. Mater. http:// dx.doi.org/10.1002/adma.201500275 (2015). 
2. Zuckermann, R. N. \& Kodadek, T. Peptoids as potential therapeutics. Curr. Opin. Mol. Ther. 11, 299-307 (2009).

3. Sun, J. \& Zuckermann, R. N. Peptoid polymers: a highly designable bioinspired material. ACS Nano 7, 4715-4732 (2013).

4. Butterfoss, G. L. et al. De novo structure prediction and experimental characterization of folded peptoid oligomers. Proc. Natl Acad. Sci. USA 109, 14320-14325 (2012).

5. Yoo, B. \& Kirshenbaum, K. Peptoid architectures: elaboration, actuation, and application. Curr. Opin. Chem. Biol. 12, 714-721 (2008)

6. Laursen, J. S., Engel-Andreasen, J., Fristrup, P., Harris, P. \& Olsen, C. A. Cis-trans amide bond rotamers in $\beta$-peptoids and peptoids: evaluation of stereoelectronic effects in backbone and side chains. J. Am. Chem. Soc. 135, 2835-2844 (2013).

7. Butterfoss, G. L., Renfrew, P. D., Kuhlman, B., Kirshenbaum, K. \& Bonneau, R. A preliminary survey of the peptoid folding landscape. J. Am. Chem. Soc. 131, 16798-16807 (2009).

8. Nam, K. T. et al. Free-floating ultrathin two-dimensional crystals from sequencespecific peptoid polymers. Nature Mater. 9, 454-460 (2010).

9. Drexler, K. E. Peptoids at the 7th Summit: toward macromolecular systems engineering. Biopolymers 96, 537-544 (2011).

10. Sanii, B. et al. Shaken, not stirred: collapsing a peptoid monolayer to produce freefloating, stable nanosheets. J. Am. Chem. Soc. 133, 20808-20815 (2011).

11. Kudirka, R. et al. Folding of a single-chain, information-rich polypeptoid sequence into a highly ordered nanosheet. Biopolymers 96, 586-595 (2011).

12. Sanii, B. et al. Structure-determining step in the hierarchical assembly of peptoid nanosheets. ACS Nano 8, 11674-11684 (2014).

13. Robertson, E. J. et al. Assembly and molecular order of two-dimensional peptoid nanosheets through the oil-water interface. Proc. Natl Acad. Sci. USA 111, 13284-13289 (2014).

14. Brooks, B. R. et al. CHARMM: the biomolecular simulation program. J. Comput. Chem. 30, 1545-1614 (2009)

15. Mirijanian, D. T., Mannige, R. V., Zuckermann, R. N. \& Whitelam, S. Development and use of an atomistic CHARMM-based forcefield for peptoid simulation. J. Comput. Chem. 35, 360-370 (2014).

16. Karplus, M. \& McCammon, J. A. The internal dynamics of globular proteins. CRC Crit. Rev. Biochem. 9, 293-349 (1981).

17. Halle, B. Flexibility and packing in proteins. Proc. Natl Acad. Sci. USA 99, 1274-1279 (2002).

18. Berg, J. M., Tymoczko, J. L. \& Stryer, L. Biochemistry, International Edition 7th edn (WH Freeman \& Co., 2010).
19. Hammer, N. D., Wang, X., McGuffie, B. A. \& Chapman, M. R. Amyloids: friend or foe? J. Alzheimers Dis. 13, 407-419 (2008).

20. Knowles, T. P., Oppenheim, T. W., Buell, A. K., Chirgadze, D. Y. \& Welland, M. E. Nanostructured films from hierarchical self-assembly of amyloidogenic proteins. Nature Nanotechnol. 5, 204-207 (2010)

21. Sun, J., Teran, A. A., Liao, X., Balsara, N. P. \& Zuckermann, R. N. Crystallization in sequence-defined peptoid diblock copolymers induced by microphase separation. J. Am. Chem. Soc. 136, 2070-2077 (2014).

22. Maulucci, N. et al. Synthesis, structures, and properties of nine-, twelve-, and eighteen-membered $\mathrm{N}$-benzyloxyethyl cyclic $\alpha$-peptoids. Chem. Commun. 33, 3927-3929 (2008)

23. Zhang, W. et al. PEG-stabilized bilayer nanodisks as carriers for doxorubicin delivery. Mol. Pharm. 11, 3279-3290 (2014).

Supplementary Information is available in the online version of the paper.

Acknowledgements Portions of this work were done as a User project at the Molecular Foundry at Lawrence Berkeley National Laboratory, supported by the Office of Science, Office of Basic Energy Sciences, of the US Department of Energy under contract no. DE-AC02-05CH1 1231. R.V.M., T.K.H., C.P., E.J.R., A.B., R.N.Z. and S.W. were supported by the Defense Threat Reduction Agency under contract no. IACRO-B0845281. C.P. was also supported by the Natural Sciences and Engineering Research Council of Canada (NSERC PDF). R.N.Z. and S.W. were also supported by the Office of Science, Office of Basic Energy Sciences, of the US Department of Energy under contract no. DE-AC02-05CH1 1231. We thank G. K. Olivier for providing the AFM data. This work used resources of the National Energy Research Scientific Computing Center, which is supported by the Office of Science of the US Department of Energy under contract no. DE-AC02-05CH11231. Quantum-mechanical calculations were carried out on the High Performance Computing resources at New York University Abu Dhabi.

Author Contributions R.V.M., R.N.Z. and S.W. initiated the research. R.V.M. and S.W. designed the molecular-dynamics simulations; R.V.M. performed the simulations. T.K.H. performed simulated X-ray-scattering calculations; C.P., E.J.R. and A.B. performed the experiments; G.L.B. designed and performed the quantum-mechanical calculations. All authors contributed to analysing the results and writing the paper.

Author Information Reprints and permissions information is available at www.nature.com/reprints. The authors declare no competing financial interests. Readers are welcome to comment on the online version of the paper. Correspondence and requests for materials should be addressed to R.V.M. (rvmannige@lbl.gov) or S.W. (swhitelam@lbl.gov). 


\section{METHODS}

Experimental nanosheet synthesis. Block-charge peptoids of lengths $L=4,8,12$, 16 or 28 residues were synthesized and purified using automated solid-phase synthesis ${ }^{3}$. For $L \geq 12$ residues (Supplementary Fig. 18d-f) and $L>12$ residues (Fig. 3e), peptoids were present at a concentration of $20 \mu \mathrm{M}$ in $10 \mathrm{mM}$ Tris buffer $\mathrm{pH}$ 8.0. Peptoid nanosheets of length $L \leq 12$ were absent at this concentration (Supplementary Fig. 18d), perhaps because not enough polymer adsorbed to the air-water interface (see discussions following Supplementary Fig. 18d); therefore we attempted to produce $L \leq 12$ nanosheets at higher concentrations (Fig. $3 \mathrm{e}$ and Supplementary Fig. 18a-c). Peptoids of length $L=12$ were present at a concentration of $1 \mathrm{mM}$ (Supplementary Fig. 18c), of length $L=8$ at $22 \mathrm{mM}$ (Supplementary Fig. 18b), and of length $L=4$ at $41 \mathrm{mM}$ (Supplementary Fig. 18a), all in $10 \mathrm{mM}$ Tris buffer, $\mathrm{pH}$ 8.0. Sheets were prepared by agitation via the vial-rocking method $^{10}$. To test whether the peptoids were adsorbing to the air-water interfacea prerequisite to assembly-we obtained surface tension data for the $L=4,8$ and 12 peptoids via the pendant drop method (see discussions following Supplementary Fig. 18) ${ }^{24}$

Nanosheet yield analysis by optical microscopy. Identical volumes were removed from each sample and applied to a thin agarose-gel slice to allow imaging for Fig. 3e and Supplementary Fig. 18 (ref. 25).

Measuring distances by single-angle $\mathrm{X}$-ray scattering and AFM. Nanosheet thicknesses were calculated via $\mathrm{AFM}^{8,10,13}$. We obtained AFM images of dry nanosheets deposited on a mica substrate in ambient air. Other distances, such as intrapolymer spacings (Fig. 2 and Supplementary Fig. 8), were obtained via $\mathrm{XRD}^{8,10,11,13}$. XRD data also show a peak corresponding to the thickness of the nanosheet.

Molecular-dynamics protocol. We surveyed a range of low-energy nanosheet configurations (Supplementary Fig. 4a) as starting points for molecular-dynamics simulations. To conduct this survey we built 28 -residue polymers into monolayers in a brick-like arrangement, and joined two identical opposing monolayers to form a bilayer (Supplementary Fig. 4a). The brick arrangement is suggested by simple electrostatic considerations, because peptoids' charged sidechains are segregated into positive and negative blocks along each chain ${ }^{11}$ (Fig. 1a). The computational search space so defined possesses six degrees of freedom, described by distances between polymers within the same layer and between monolayers (Supplementary Fig. 4a). We calculated energies (in implicit water) for a large number of these parameter sets, and chose six low-energy versions on which to focus (Supplementary Fig. 4b). Each nanosheet version was then explicitly solvated and relaxed in a sequence of protocols (see Supplementary Fig. 4d), before undergoing constant-pressure molecular-dynamics simulation using a leap-frog integrator (a commonly used second-order numerical method for integrating equations of motion) with a $1-\mathrm{fs}$ timestep.

Each of the six low-energy versions of the nanosheet was seen to be stable for over $50 \mathrm{~ns}$ of molecular-dynamics simulation at standard temperature and pressure; that is, each had ceased to evolve (structural snapshots for each simulation are available in Supplementary Information), and did not dissolve or convert to a different structure (Supplementary Fig. 5). (In contrast, high-energy initial configurations did not yield stable structures.) These simulations reveal that, in the region of the energy minimum dictated by charged sidechains ${ }^{11}$, there exists a rugged free-energy landscape with a range of near-degenerate nanosheet structures. Biomolecules, by contrast, often possess clear free-energy minima ${ }^{26-30}$

Our simulations represent periodically replicated patches of nanosheets of approximate dimensions $60 \mathrm{~nm} \times 18 \mathrm{~nm}$, and so do not address the nature of nanosheet order on the micrometre scale. Given that nanosheets are produced by a far-from-equilibrium mechanical protocol ${ }^{10}$, and given the stability of all six nanosheet versions in our simulations, it is possible that extended nanosheets consist of a patchwork of different types of stable local order. Nonetheless, each of the six low-energy nanosheet versions displays molecular features consistent with experiments (Supplementary Fig. 8), from which we infer that substantial portions of nanosheets display locally the atomic-scale features seen in our simulations. In the main text, for brevity, we present results from one particular nanosheet version, number 5 .

Supplementary Figure 4 describes our generation of the six molecular-dynamics starting configurations. Molecular-dynamics simulations were done using the CHARMM software package ${ }^{14}$. Aside from the initial set-up, all nanosheet series were simulated using a leap-frog integration algorithm with a 1-fs timestep. Simulations were performed in the isothermal-isobaric (NPT, for constant particle number, pressure and temperature) ensemble at $300 \mathrm{~K}$ and 1 atmospheric pressure, in an orthorhombic periodic box in which the three orthogonal box dimensions were allowed to vary independently. The Hoover algorithm was used to maintain constant pressure ${ }^{31}$. Hydrogen bonds were constrained using the SHAKE protocol ${ }^{32}$. Particle-mesh Ewald summation was used to evaluate longrange electrostatic interactions, with a real-space cut-off of $12 \AA$, a sixth-order cubic spline, and a $\kappa$ value (width of the Gaussian distribution) of 0.34 . Van der Waals interactions were calculated up to a distance of $12 \AA$, with a smoothing function applied from $10 \AA$ to $12 \AA$.

Simulations reported in the main text were performed in a solution consisting of water, the nanosheet's counterions, and potassium chloride at a concentration of $10 \mathrm{mM}$. We also carried out a series of independent simulations of the version 5 nanosheet, in which potassium chloride concentrations were set to $0.1 \mathrm{mM}, 1 \mathrm{mM}$ $50 \mathrm{mM}$ and $100 \mathrm{mM}$ (Supplementary Fig. 14). These simulations verified that simulated nanosheet structures do not change markedly over the range of salt concentrations used in our experiments ${ }^{8,10,11}$.

Computational X-ray scattering. We calculated scattering spectra at wavevector $\boldsymbol{q}$ using the expression

$$
I(\boldsymbol{q})=\left|\sum_{j} f_{j} \exp \left(i \boldsymbol{q} \cdot \boldsymbol{r}_{j}\right)\right|^{2}
$$

where the sum runs over all atoms, $f_{j}$ is the atomic scattering factor for atom $j$ and $\boldsymbol{r}_{j}$ is the position of atom $j$. Equation (1) assumes that electrons are localized at atomic sites. We let $f_{j}$ equal the atomic number, an approximation that is nearly exact at the experimental X-ray energies of $11 \mathrm{keV}$ (ref. 33). The values of $\boldsymbol{q}$ were discretized so as to be commensurate with the periodic box.

Experimental in-plane X-ray-scattering spectra were taken by allowing nanosheets to dry on a Kapton grid, stacking the sheets on top of each other to produce a sample with a uniform orientation ${ }^{12}$. The absence of lamellar peaks when the X-ray beam was fired 'face-on' into the stack confirmed that the nanosheet normals were uniformly oriented parallel to the grid normal. However, the radially averaged signal indicated that various sheets and/or domains existed within the beamline, as expected given that the beam cross-section $(120 \mu \mathrm{m} \times 800 \mu \mathrm{m})$ is greater than the typical nanosheet size $(20 \mu \mathrm{m} \times 20 \mu \mathrm{m})$. To compare with these in-plane, radially averaged spectra, we radially averaged the simulated scattering spectra (equation (1)) in the $x y$ plane of the nanosheet, $I\left(q_{x y}\right)=\langle I(\boldsymbol{q})\rangle_{q_{z}=0, \sqrt{q_{x}^{2}+q_{y}^{2}}=q_{x y}}$. Finally, these intensities $\left(I\left(q_{x y}\right)\right)$ were normalized by the expected intensity of an equivalent ideal gas $\left(I_{\mathrm{IG}}\left(q_{x y}\right)\right)$, which is discussed in Supplementary Fig. 17.

Direct measurements. While X-ray scattering is useful for comparing simulation to experiment, direct measurements of some features (for example, polymer $y$-padding, nearest neighbour $\mathrm{N}-\mathrm{N}$ spacing, bilayer thickness) provide additional information with regards to distances and distributions. Supplementary Fig. 7 and its associated text discusses those measurements and how they are calculated, while Supplementary Fig. 8 compares experimental observations to those metrics. Measurement of order in the bilayer. For each peptoid (Fig. 3a), we designate the position of the backbone nitrogen of the $i$ th residue to be $\boldsymbol{r}_{i}$. A simple measure of order is the angle $\theta_{\text {backbone, } x}$ between each peptoid backbone and the $x$ axis

$$
\theta_{\text {backbone }, x}=\cos ^{-1}\left[\frac{\left(\boldsymbol{r}_{\text {last }}-\boldsymbol{r}_{\text {second }}\right) \cdot \hat{\boldsymbol{x}}}{\left|\boldsymbol{r}_{\text {last }}-\boldsymbol{r}_{\text {second }}\right|}\right]
$$

$\alpha$-Helical and $\beta$-sheet segments from proteins. A structure database was obtained from the Structural Classification of Proteins (SCOPe; release 2.03) that contains proteins with no more than $40 \%$ sequence identity with each other (http://scop. berkeley.edu/downloads/pdbstyle/pdbstyle-sel-gs-bib-40-2.03.tgz). $\alpha$-Helical and $\beta$-sheet segments were identified using the DSSP (Define Secondary Structure of Proteins) algorithm ${ }^{34} .(\phi, \psi)$ pairs were obtained for all residues within $\alpha$-helical and $\beta$-sheet segments, which contributed towards the histograms in Fig. $4 b, d$, respectively

Quantum mechanics calculations. Density functional theory was used to assess the low-energy structures available to a peptoid backbone consisting of two alternating sets of dihedral angles $(\phi, \psi)$. B3LYP and M05-2X were the two functionals used to calculate the energies of a configuration. B3LYP is a commonly used and widely applicable functional ${ }^{35}$, and M05-2X is a newer functional that accounts for dispersion forces ${ }^{22}$. Gaussian 09 (C.01) was used for density functional theory calculations ${ }^{23}$. For each peptoid model, the alternating pattern is defined by the numbers $A$ and $B$ (Fig. $4 \mathrm{~h})$, where adjacent $(\phi, \psi)$ pairs take the values $(-A, B)$ and $(-B, A)$. $A$ and $B$ were constrained to range from $50^{\circ}$ to $180^{\circ}$ in steps of $10^{\circ}$. Molecules were optimized at the HF/6-31G* level of theory ${ }^{36}$, with $A$ and $B$ constrained and all other degrees of freedom unconstrained. The lowest energy associated with each $(A, B)$ pair is reported in the Ramachandran plots of Fig. $4 \mathrm{~h}$ and Supplementary Figs 24-26.

24. Rosen, M. J. \& Kunjappu, J. T. Surfactants and Interfacial Phenomena (John Wiley \& Sons, 2012)

25. Leopold, P. E., Montal, M. \& Onuchic, J. N. Protein folding funnels: a kinetic approach to the sequence-structure relationship. Proc. Natl Acad. Sci. USA $\mathbf{8 9}$, 8721-8725 (1992) 


\section{RESEARCH LETTER}

26. Bryngelson, J. D., Onuchic, J. N., Socci, N. D. \& Wolynes, P. G. Funnels, pathways, and the energy landscape of protein folding: a synthesis. Proteins $21,167-195$ (1995).

27. Schug, A. \& Onuchic, J. N. From protein folding to protein function and biomolecular binding by energy landscape theory. Curr. Opin. Pharmacol. 10, 709-714 (2010).

28. Shan, Y. et al. How does a drug molecule find its target binding site? J. Am. Chem Soc. 133, 9181-9183 (2011)

29. Hoover, W. G. Canonical dynamics: equilibrium phase-space distributions. Phys. Rev. A 31, 1695-1697 (1985)

30. Allen, M. P. \& Tildesley, D. J. Computer Simulation of Liquids (Oxford Univ. Press, 1989).
31. Chantler, $C$. Theoretical form factor, attenuation, and scattering tabulation for $Z=$ 1-92 from $E=1-10$ eV to $E=0.4-1.0 \mathrm{MeV}$. J. Phys. Chem. Ref. Data 24, 71 (1995).

32. Kabsch, W. \& Sander, C. Dictionary of protein secondary structure: pattern recognition of hydrogen-bonded and geometrical features. Biopolymers 22 2577-2637 (1983)

33. Lee, C., Yang, W. \& Parr, R. G. Development of the Colle-Salvetti correlation-energy formula into a functional of the electron density. Phys. Rev. B 37, 785-789 (1988)

34. Zhao, Y., Schultz, N. E. \& Truhlar, D. G. Exchange-correlation functional with broad accuracy for metallic and nonmetallic compounds, kinetics, and noncovalent interactions. J. Chem. Phys. 123, 161103 (2005)

35. Frisch, M. J. et al. Gaussian09 Revision D.01 (Gaussian Inc., Wallingford, 2009).

36. Roothaan, C. C. J. New developments in molecular orbital theory. Rev. Mod. Phys. 23, 69-99 (1951). 